\title{
Breast cancer detection using combined curvelet based enhancement and a novel segmentation methods
}

\author{
Balasubramaniam Senthilkumar, Govindaswamy Umamaheswari
}

\begin{abstract}
Aim. This paper describes the digital implementation of a mathematical transform namely 2D Fast Discrete Curvelet Transform (FDCT) via UnequiSpaced Fast Fourier Transform (USFFT) in combination with the novel segmentation method for effective detection of breast cancer.

Methods. USFFT performs exact reconstructions with high image clarity. Radon, ridgelet and Cartesian filters are included in this method. Mean Square Error (MSE) and Peak Signal to Noise Ratio (PSNR) were calculated for the image and the resulting value showed that the proposed method performs well on mammogram image in reducing noise with good extraction of edges. This work includes a novel segmentation method, which combines Modified Local Range Modification (MLRM) and Laplacian of Gaussian (LoG) edge detection method to segment the textured features in the mammogram image.
\end{abstract}

Results. The result was analyzed using a Receiver Operating Characteristics (ROC) plot and the detection accuracy found was $99 \%$ which is good compared to existing methods.

Key words: USFFT, breast cancer, mammogram, MLRM, LoG

Received: July 13, 2013; Accepted: December 19, 2013; Available online: January 23, 2014 http://dx.doi.org/10.5507/bp.2013.097

Department of Electronics and Communication Engineering, PSG College of Technology, Coimbatore, Tamilnadu, India 641004 Corresponding author: Balasubramaniam Senthilkumar, e-mail:senthilvlsi@yahoo.com

\section{INTRODUCTION}

Breast cancer is a major cause for more percentage of cancer mortality in both developing and developed countries. It has been shown that early detection and treatment are the most effective methods for reducing the mortality. Microcalcification is the main sign of malignancy. Detection of small malignancies is especially difficult in younger women who tend to present denser breast tissue. Digital mammography is a widely used aid in the detection of breast cancer. However, mammographic images are inherently noisy and usually contain low contrast regions ${ }^{1}$. Noise presents in digital mammograms because of artifacts like dust, hair, capturing and storing. Dense regions in digital mammographic images are usually noisy and have low contrast. For this reason, detection and diagnosis of a cancerous area is a tough task for the radiologist. It is also necessary to remove the noise before processing the mammogram. Over the last two decades, many noise removal algorithms have been proposed. There has also been abundant interest in wavelet methods for noise removal in mammogram images. Initial efforts included very simple ideas like thresholding of the orthogonal wavelet coefficient, tree based wavelet denoising, level dependent thresholding, block thresholding, adaptive thresholding, discrete ridgelet and curvelet transforms as an alternate for wavelet and for the representation of both smooth objects and objects with edges. Wavelet shrinkage based noise removal was proposed by ${ }^{1}$. Local contrast modification was tested $b^{2}$. Multiscale edge enhancement was proposed $b^{3}$, ridgelet and curvelet methods have been tested in ${ }^{4}$ and Fast Discrete Curvelet Transform (FDCT) via wedge wrapping has been tested by $^{5}$. The work described here tested another FDCT via USFFT for effective noise removal and edge preservation with a new segmentation (MLRM and LoG) for breast cancer detection.

\section{MATERIAL AND METHODS}

The database of mammograms used in this work is known as Mammographic Image Analysis Society (MIAS) Mini Mammographic Database. The example image used in this paper is mdb075. The entire method presented was implemented in MATLAB 7.0. Image Processing and Wavelet Toolboxes was used for this work. The methodology used consists of two main stages. First is the construction of FDCT via USFFT for noise removal and the second is the segmentation (combination of MLRM and LoG) stage.

\section{Noise removal}

Curvelet transform was designed to represent edges and other singularities along curves much more efficiently than traditional transforms. Many wavelet coefficients are required to reconstruct the edges in an image properly. Because of this, wavelets are not able to produce accurate results. Ridgelet transforms provide a sparse representation of both smooth functions and of perfectly straight edges. In image processing, edges are curved rather than straight and ridgelets alone cannot yield efficient repre- 
sentations. Hence, curvelet (ridgelet in multiscale) is used. The USFFT version uses a decimated rectangular grid tilted along the main direction of each curvelet and for the digital transform tilting the grids induces a resampling of the Fourier transform on semi regular grids. For the inversion, a conjugate gradient solver rapidly converges to the solution. The procedure consists of: oversampling Cartesian grid by zero padding, evaluation of 2D Fast Fourier Transform (FFT) of the function, association of Cartesian neighbours to each grid point, approximation of interpolation to get the Cartesian grid values and perform the Cartesian 2D inverse FFT. Oversampling and interpolation are done by Euler sum or Langrangian interpolation, the fast multipole method or oversampling and obtaining the partial derivatives and then interpolating by Taylor series. Curvelet in the frequency domain has been referred from ${ }^{6}$ and can be written as $\varphi_{j, l, k}(\xi)=W_{j}\left(R_{\theta_{j, l}} \xi\right) e^{i\left(k, R_{\left.\theta_{j, l}, \xi\right)}\right.}, k \in \wedge$ where $\wedge=2^{-j} \times 2^{-j / 2}$. Cartesian grid and $W_{j}(\xi)=w\left(2^{-j}|\xi|\right) v\left(2^{\lfloor j / 2\rfloor} \theta\right)$.

The coefficients are given by $\theta_{j, l, k}(f)=\int \hat{f}(\xi) W_{j, 0}\left(R_{\theta_{l}} \xi\right) e^{-i\left(R_{\theta_{l}}^{-1, k}, \xi\right)} d \xi$ the Cartesian equivalent is given by, $\theta_{j, l, k}(f)=\int \hat{f}(\xi) W_{j, 0}\left(S_{\theta_{l}} \xi\right) e^{-i\left(S_{\theta_{l}}^{-1, k}\right)} d \xi, S_{\theta_{l}}=\left[\begin{array}{cc}1 & 0 \\ -\tan \theta & 1\end{array}\right]$, to calculate the discrete curvelet coefficients, assume that window $W_{j 0}\left(n_{1}, n_{2}\right)$ is supported within a shifted rectangle $P_{j}=\left\{\left(n_{1}, n_{2}\right): 0 \leq n_{1}-n_{0}<L_{j},-l_{j} / 2 \leq n_{2}<l_{j} / 2\right\}$. Discrete curvelet coefficients are given by $\theta_{j, l, k}^{D}=\sum_{n_{1}, n_{2} \in P_{j}} \hat{f}\left(n_{1}, n_{2}+n_{1} \tan \theta_{j . l}\right) W_{j 0}\left(n_{1}, n_{2}\right) e^{-2 \pi\left(n_{1} k_{1} / L_{j}+n_{2} k_{2} / L_{j}\right)}$ here $\left(n_{1}, n_{2}\right)$ is the Cartesian grid and $\left(n_{2}, n_{1} \tan \theta\right)$ is the sheared grid. The steps for obtaining the coefficients are as follows. The Cartesian array $f\left(i_{1}, i_{2}\right) \quad 0 \leq i_{1}, i_{2} \leq N-1$.

1. Apply the 2D FFT and obtain the Fourier samples $f\left(n_{1}, n_{2}\right)-N / 2 \leq n_{1}, \quad n_{2}<N / 2$.

2. For each scale /angle pair $(j, 1)$, interpolate the object to obtain sampled values in the parallelepiped $P_{j, 1}=\left\{\left(n_{1}, n_{2}+n_{1} \tan \theta_{j, 1}\right)\right\}, n_{1,} n_{2} \in P_{j}$
3. Multiply the interpolated/sheared object $\hat{f}$ near the parallelepiped with orientation $\theta_{j, 1}$ and obtain $\hat{f}\left(n_{1}, n_{2}+n_{1} \tan \theta_{j, l}\right) W_{j 0}\left(n_{1}, n_{2}\right) e^{-2 \pi\left(n_{1} k_{1} / L_{j}+n_{2} k_{2} / L_{j}\right)}$

4. Apply the inverse 2D FFT to each $f_{j, l}$ hence collecting the discrete coefficients $\theta_{j, l, k}^{D}$.

After applying the inverse 2D FFT, the coefficients are collected and the image has been reconstructed. The performance of this method was tested by calculating two important error metrics. They are, Mean Square Error (MSE) and Peak Signal to Noise Ratio (PSNR). The calculated values of both error metrics are compared with the existing noise reduction techniques and tabulated in Table 1. The MSE is greatly reduced and the PSNR is increased accordingly for the proposed method. From this, it is noted that, the proposed method works well on mammogram image for noise reduction.

\section{Segmentation}

The edge detection process involves in finding the exact breast area for segmentation. It also plays the main role in finding asymmetry between the breasts. This can be achieved by detecting the nipple position and with respect to the position, the breasts are compared to find the asymmetry. There are many existing edge detection methods like Roberts, Sobel, Prewitt, Laplacian of Gaussian. The edge detected image can be easily segmented for observing a Region of Interest (RoI). Here a new method of edge detection based segmentation was tested. This work combined the Modified Local Range Modification (MLRM) contrast enhancement method $^{7}$ with Laplacian of Gaussian (LoG) edge detection method for the segmentation of a RoI. This new combination effectively segments the microcalcification clusters and textured features. The suspected area in the mammogram image was detected from the shape of the segmented clusters.

Table 1. MSE and PSNR comparison of proposed method with the existing methods.

\begin{tabular}{lrrrrrr}
\hline \multirow{2}{*}{ Techniques } & \multicolumn{2}{c}{ Mdb075 } & \multicolumn{2}{c}{ Mdb072 } & \multicolumn{2}{c}{ Mdb002 } \\
\cline { 2 - 6 } & MSE & PSNR & MSE & PSNR & MSE & PSNR \\
\hline Without Noise Removal & 396.11 & 22.15 & 397.56 & 22.14 & 391.34 & 22.21 \\
HAAR & 204.76 & 25.02 & 205.97 & 24.99 & 201.15 & 25.10 \\
Daubechies 4 & 154.17 & 26.25 & 156.81 & 26.18 & 142.78 & 26.58 \\
FDCT - wedge wrapping & 29.52 & 33.43 & 34.93 & 32.70 & 25.62 & 34.05 \\
Proposed (FDCT - USFFT) & 7.43 & 39.42 & 9.16 & 38.51 & 6.78 & 39.82 \\
\hline
\end{tabular}

Table 2. Detection Accuracy comparison of proposed method with the existing methods.

\begin{tabular}{lc}
\hline Main Techniques involved in Breast Cancer Detection & Detection Accuracy \\
\hline Entropic Thresholding (ET) $\left(\right.$ ref. $^{8}$ ) & $86 \%$ \\
Wavelet and Neural Network (WNN) (ref. ${ }^{9}$ ) & $88 \%$ \\
Bilateral Image Subtraction (BIS) $\left(\right.$ ref. $\left.^{10}\right)$ & $95 \%$ \\
Tree Structured Wavelet Transform filter with Neural Network (TSWTNN) (ref. $\left.{ }^{11}\right)$ & $97 \%$ \\
Proposed Method (MLRM and LoG) & $99 \%$ \\
\hline
\end{tabular}


Comparison of proposed method (MLRM and LoG) with existing methods is tabulated in Table 2. The detection accuracy was found based on the detection of Malignant (cancerous) and Benign (non cancerous) abnormalities.

\section{RESULTS}

The sample mammogram image used in this work was mdb075. This had already been detected as cancerous images (malignancy) by the radiologists and had also been certified by the MIAS database. The proposed method which also found the same result and segmented the exact cancer (textured feature) location with good clarity is shown in Fig. 1c. Fig. 1a is the original image, Fig. 1b is the USFFT filtered image and Fig. 1c is the cancer segmented image.

\section{DISCUSSION}

Fig. 1a shows the original mammogram image mdb075. From the original image, the microcalcification cluster is not clearly outlined because it is a fatty and dense breast but after wavelet based USFFT filtering, the MSE was greatly reduced and the PSNR was increased accordingly compared to existing methods shown in Table 1. This enhancement method eliminated the unwanted noises from mammogram image and enhanced the edges with good clarity as shown in Fig. 1b. It is clear that the image contains cluster (texture feature) of microcalcification in dense tissues after applying the proposed segmentation shown in Fig. 1c. This new approach not only detects the microcalcification clusters, it is also involved in detecting the shape of the microcalcification cluster. The malignant and benign clusters were detected on the basis of shape. 126 sample images were tested in this work. Statistical moments were calculated on the basis of the intensity histogram for the detection of malignant and benign abnormalities. Further, the Receiver Operating Characteristics (ROC) analysis has been done and the curve was plotted for calculated True Positive Fraction/sensitivity (TPF) and False Positive Fraction/specificity (FPF) as shown in Fig. 2. The average ROC values of proposed method was compared with existing techniques and tabulated in Table 2. The results were superior in detecting cancer (malignancy) with an accuracy of $99 \%$, which is much more efficient than the other existing methods.

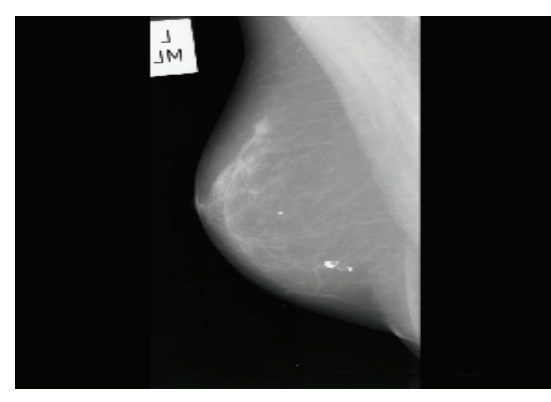

(a) Original Image

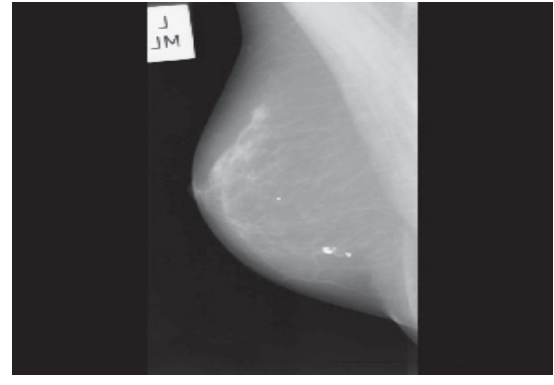

(b) Proposed Curvelet Filtered Image

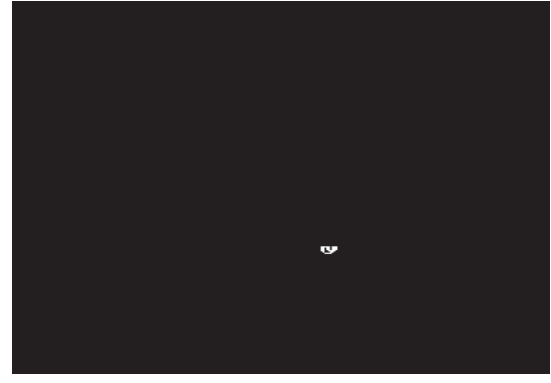

(c) MLRM and LoG Segmented Image

Fig 1. Mammogram Image mdb075 (Referred from MIAS database).

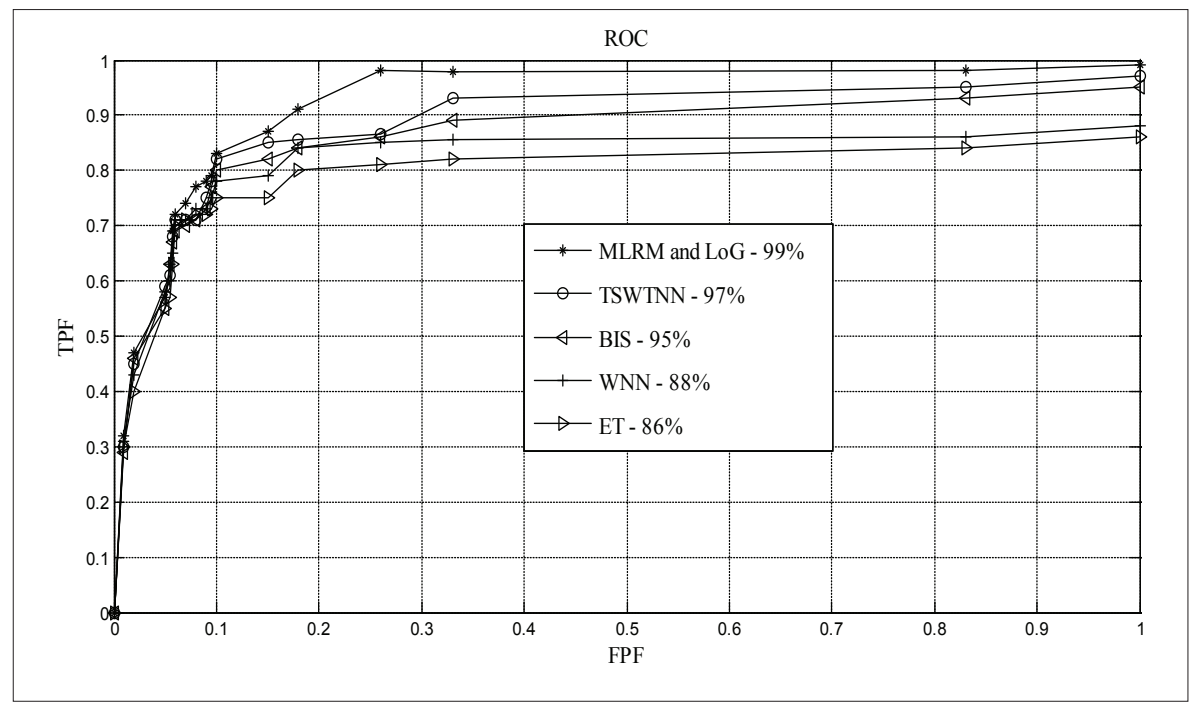

Fig. 2. ROC of the proposed method. 


\section{CONCLUSION}

Digital implementation of 2D FDCT via USFFT has been done to reduce the noise and enhance the digital mammogram image. MSE and PSNR were calculated and compared with existing enhancement methods. The resulting values show that the proposed method performs well on mammogram image in reducing noise with good extraction of edges. This work also includes a new segmentation method which combines MLRM and LoG for the segmentation of textured features in mammogram image. The ROC was plotted and the detection accuracy found was $99 \%$ which is better than existing methods. The computational complexity of the proposed method is little bit higher. In future, it is planned to reduce this and to modify it as automated method for effective breast cancer detection.

\section{ABBREVIATIONS}

BIS, Bilateral Image Subtraction; ET, Entropic Thresholding; FDCT, Fast Discrete Curvelet Transform; FPF, False Positive Fraction; FFT, Fast Fourier Transform; LoG, Laplacian of Gaussian; MIAS, Mammographic Image Analysis Society; MLRM, Modified Local Range Modification; MSE, Mean Square Error; PSNR, Peak Signal to Noise Ratio; ROC, Receiver Operating Characteristics; TPF, True Positive Fraction; TSWTNN, Tree Structured Wavelet Transform filter with Neural Network; USFFT, UnequiSpaced Fast Fourier Transform; WNN, Wavelet and Neural Network.

\section{ACKNOWLEDGEMENT}

Conflict of interest statement: None declared.

\section{REFERENCES}

1. Scharcanski J, Jung CR. Denoising and enhancing digital mammographic images for visual screening. Computerized Medical Imaging and Graphics 2006;30(4):243-54

2. Adel M, Zuwala D, Rasigni M, Bourennane S. Filtering noise on mammographic phantom images using local contrast modification functions. Image and Vision Computing 2008;26(9):1219-29.

3. Starck JL, Murtagh F, Candes EJ, Donoho DL. Gray and Color Image Contrast Enhancement by the Curvelet Transform. IEEE Transactions on Image Processing 2003;12(6):706-17.

4. Starck JL, Candes EJ, Donoho DL. The Curvelet Transform for Image Denoising. IEEE Transactions on Image Processing 2002;11(6):670 84.

5. Patil AA, Singhai J. Image denoising using Curvelet transform: an approach for edge preservation. Journal of Scientific \& Industrial Research 2010;69(1):34-8.

6. Soman KP, Ramachandran K I. Insight into Wavelets. India: Prentice - Hall India; 2005.

7. Senthilkumar B, Umamaheswari G. Novel Preprocessing Technique in the Computer Aided Detection of Breast Cancer. Journal of Computer Science 2012;8(12):1957-60.

8. Rojas Dominguez A, Nandi AK. Detection of masses in mammograms via statistically based enhancement, multilevel-thresholding segmentation and region selection. Computerized Medical Imaging and Graphics 2008;32(4):304-15. doi: 10.1016/j.compmedimag.2008.01.006

9. Retico A, Delogu P, Fantacci ME, Preite-Martinez A, Stefanini A, Tata A. A scalable computer - aided detection system for microcalcification cluster identification in a pan - European distributed database of mammograms. Nuclear Instruments and Methods in Physics Research 2006;569(2):601-05. doi:10.1016/j.nima.2006.08.094

10. Cheng HD, Shi XJ, Min R, Hu LM, Cai XP, Du HN. Approaches for Automated Detection and Classification of Masses in Mammograms. Pattern Recognition 2006; 39(4):646-68.

11. Zhang L, Shankar R, Qian W. Advances in micro - calcification clusters detection in mammography. Computers in Biology and Medicine 2002;32(6):515-28. 